\title{
Liver mass containing normal bile ducts in an Alagille patient: A case report
}

\author{
Stefanie Parisien-La Salle, Med. Int. ${ }^{a}$, Justin Côté-Daigneault, M.D. ${ }^{b}$, Laurent Garel, M.D. ${ }^{c}$, \\ Luc Laurier Oligny, M.D. ${ }^{c}$ and Fernando Alvarez, M.D.
}

\begin{abstract}
This manuscript reports a case of a patient with Alagille syndrome who developed a rapidly growing lesion in the caudate segment of his cirrhotic liver. This mass was closely monitored but did not seem malignant from a radiological point of view. An MRI showed no criteria in favour of a hepatocarcinoma, however, the rapid growth lead to a biopsy of both the lesion and the cirrhotic liver. The pathology results indicated normal development of the bile ducts in the mass and paucity of the biliary ducts in the second liver specimen. We will describe this case and propose an interpretation of these findings.

Key words: Alagille syndrome, bile ducts, paucity of interlobular bile ducts, liver, caudate lobe.
\end{abstract}

http:/ /dx.doi.org/10.5546/aap.2015.eng.e223

\section{INTRODUCTION}

Alagille syndrome (AGS) is an autosomal dominant disease with variable expression that results from mutations in JAGGED1 and NOTCH $2,{ }^{1}$ which are the genes involved in the pathway associated with normal biliary development. The main feature is a paucity of the interlobular bile ducts, leading to chronic cholestasis in $91 \%$ of patients. Other major abnormalities defining this syndrome are congenital heart disease $(85 \%)$, butterfly vertebrae $(87 \%)$, embryotoxon $(88 \%)$ and characteristic facial traits $(95 \%) .{ }^{2}$ Clinical diagnosis is possible when the patient presents at least three of these major signs. ${ }^{2}$

a. University of Montreal

b. CHUM (University of Montreal Hospital Center).

c. Sainte-Justine Hospital

\section{E-mail Address:}

Stefanie Parisien-La Salle, Med. Int.

stefanie.parisien-la.salle@umontreal.ca

Funding: None

Conflict of interest: None.

Received: 1-3-2015

Accepted: 3-2-2015

\section{CASE REPORT}

At four weeks of age, the patient was hospitalized for neonatal cholestasis and pale coloured stools. His initial liver function tests showed hepatic injury and obstructive cholestasis (ALT: 74U/L [N: 5-34] AST: 154U/L [N: 11-43], gamma-GT: 81U/L [N:3-43]), and an elevated conjugated bilirubin (109umol/L [N:0-4]). AGS was diagnosed one month later due to additional clinical findings including butterfly vertebrae located in T5, characteristic facial traits, peripheral pulmonary stenosis and persistent jaundice. A liver biopsy revealed an unspecific picture of neonatal cholestasis, associated with paucity of interlobular bile ducts. The identification of JAG1 $\mathrm{r} 184 \mathrm{c}+$ mutation came four years later to confirm the initial diagnosis of a partial AGS.

At the age of 12 , the patient started presenting a liver heterogeneity; the caudate lobe became hypertrophied.

Four years later, the ultrasound showed a heterogeneous lesion measuring $6.5 \mathrm{~cm} \times 4.4 \mathrm{~cm}$, located in the caudate lobe of the liver. The diagnosis of focal nodular hyperplasia (FNH) seemed most likely at the time.

Over the following year, the mass grew in size leading to further investigations. An abdominal MRI was then performed (Figure 1). The imaging showed that the mass had reached $10.7 \mathrm{~cm} x$ $12.5 \mathrm{~cm} \times 6.9 \mathrm{~cm}$ and was well delimited. After gadolinium administration, the mass had a uniform enhancement and there were no sign of early wash out. The mass did not have a fatty content. There were no enlarged hilar lymph nodes. The spleen had reached $23 \mathrm{~cm}$ in transverse diameter, indicating the development of portal hypertension. The clinical, biochemical and radiological findings did not meet the criteria of a hepatocarcinoma. The alpha-fetoprotein remained at a normal level and a biopsy of the right and caudate lobe was performed, revealing that the mass was composed of normal liver, without ductopenia. In the sample, six out of seven portal spaces were normal and the seventh showed a subtle hepatitis with no fibrosis. The hepatocytes had a slight intracytoplasmic cholestasis that was 
described as moderate in the normal liver biopsy (Figure 2).

\section{DISCUSSION}

AGS is though to be a chronic disease with ductopenia. The mechanism underlying the bile duct paucity is not completely understood in humans. In normal cells, the JAGGED1 ligand activates the NOTCH receptors on bipotential hepatoblasts, leading to differentiation into cholangiocytes and intrahepatic bile ducts genesis. ${ }^{3}$ Spontaneous appearance of normal bile ducts is a rare phenomenon.

There seems to be an important vascular involvement in the development of abnormal ducts. Indeed, it has been shown in mice that mutating JAGGED1 in the mesenchyme of the portal vein leads to the AGS hepatic phenotype. ${ }^{4}$ This can be explained by the fact that bile ducts originate from the bi-potential cells lining the mesenchyme of the portal vein. ${ }^{5}$ In AGS, the mutated gene alters the signalisation to the
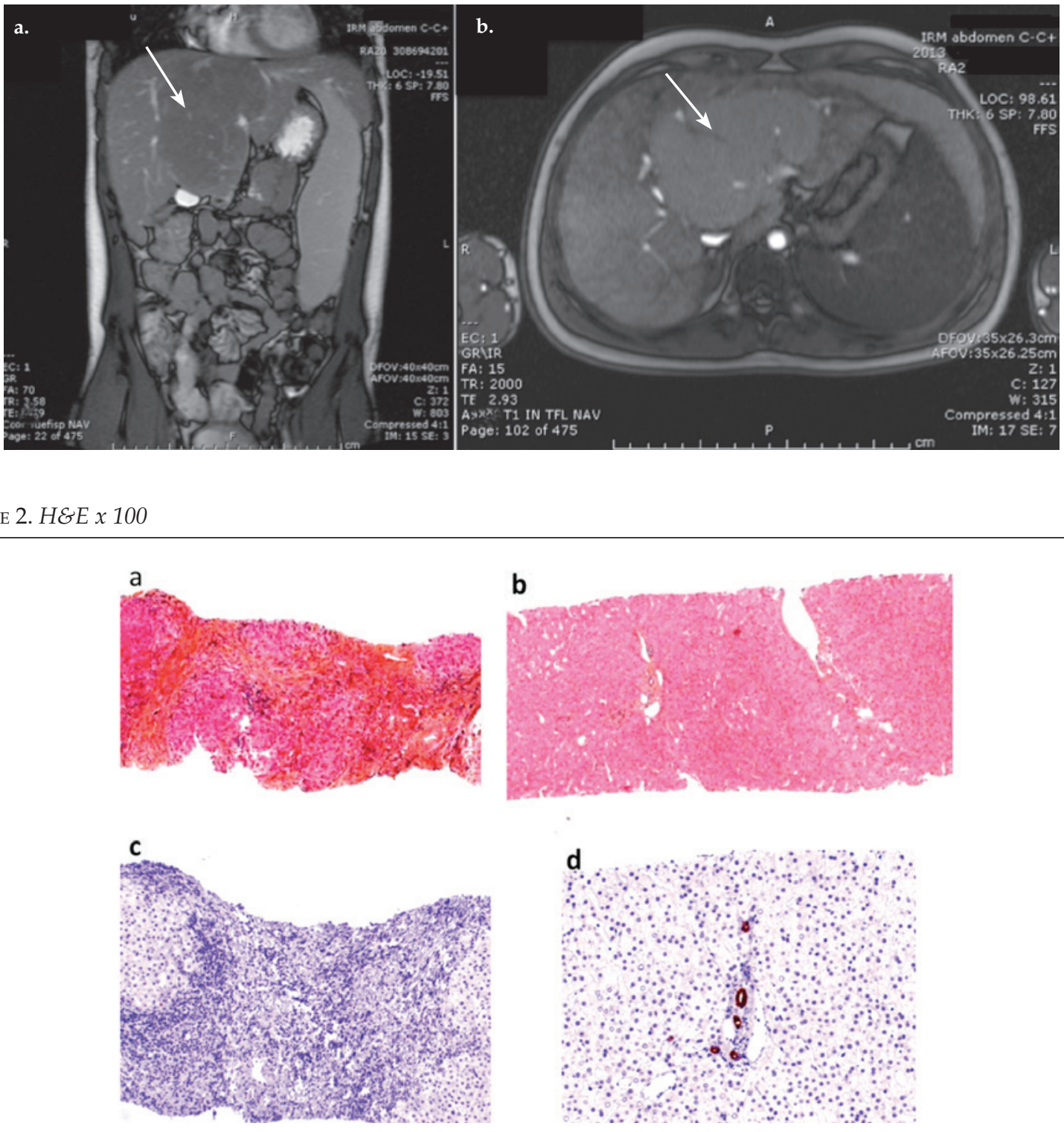

a) Extensive fibrosis, with micro-nodules in the right lobe of the liver. b) Normal liver, in the biopsy from the caudate lobe. Immunohistochemistry detecting cytokeratin 19 expression. c) Absence of interlobular bile ducts in the right lobe.

d) Presence of interlobular bile ducts in the normal liver. 
hepatoblast leading to abnormal cholangiocyte development. So, for the liver to have normal bile ducts the mutation needs to spare the cells that were destined to vascularise the caudate lobe, for example the patient could have a congenital mosaicism. This scenario is possible seeing the caudate lobe is embryonically and anatomically independent of the right and left liver and the main portal fissure. ${ }^{6}$ Another possibility is that there is an alternate pathway to ductal formation. Bile duct regeneration is theoretically possible without NOTCH signalling; however it has only been identified in murine experiments. ${ }^{3}$

The enlargement of the caudate lobe may be explained by the venous drainage of the liver. The caudate lobe drains directly into the inferior vena cava, as the rest of the liver drains into the hepatic veins first. A cirrhotic liver causes occlusion of the hepatic veins, leading to greater blood flow through the caudate lobe. Considering the particular vascular anatomy of the caudate lobe during foetal life, we can speculate that the normal JAGGED gene in this patient was only expressed in the caudate lobe allowing a normal development of bile ducts. ${ }^{7}$

In conclusion, Alagille Syndrome remains a severe disease with a $62 \%$ overall survival rate at 20 years. ${ }^{8}$ We hope this case may shed some light on the pathogenesis behind AGS and help in the better management and treatment of this disease.

\section{REFERENCES}

1. Ciocca M, Álvarez F. Síndrome de Alagille. Arch Argent Pediatr 2012;110(6):509-15.

2. Alagille D, Estara A, et al. Syndromic paucity of interlobular bile ducts (Alagille syndrome or arteriohepatic dysplasia): review of 80 cases. J Pediatr 1987;110(2):195-200.

3. Walter TJ, Vanderpool C, Cast AE, Huppert SS. Intrahepatic bile duct regeneration in mice does not require Hnf6 or Notch signaling through Rbpj. Am J Pathol 2014;184(5):1479-88.

4. Hofmann JJ, Zovein AC, Koh H, Radtke F, et al. Jagged1 in the portal vein mesenchyme regulates intrahepatic bile duct development: insights into Alagille syndrome. Development 2010;137(23):4061-72.

5. Desmet VJ. Ludwig symposium on biliary disorders--part I. Pathogenesis of ductal plate abnormalities. Mayo Clinic Proc 1998;73(1):80-9.

6. Abdalla EK, Vauthey JN, Couinaud C. The caudate lobe of the liver: implications of embryology and anatomy for surgery. Surg Oncol Clin N Am 2002;11(4):835-48.

7. Lykavieris P,Hadchouel M, ChardotC, Bernard O. Outcome of liver disease in children with Alagille syndrome: a study of 163 patients. Gut 2001;49(3):431-5.

8. Dodds WJ, EricksonSJ, Taylor AJ, Lawson TL, etal. Caudate lobe of the liver: anatomy, embryology, and pathology. AJR Am J Roentgenol 1990;154(1):87-93. 